\title{
The Effect of Hemodialysis on Axial Length, Ocular Surface, and Intraocular Pressure in Patients with End-Stage Renal Failure
}

This article was published in the following Dove Press journal: International Journal of General Medicine

\author{
Mustafa Kalayci (D) ${ }^{1,2}$ \\ Ibrahim Ali Hassan (D) \\ Ibrahim Abdi Keinan (D) \\ Ersan Cetinkaya $\mathbb{D}^{2}$ \\ Elcin Suren (I) $^{2}$ \\ Mehmet Tahtabasi (iD ${ }^{3}$ \\ Hilmi Erdem Sumbul (D) ${ }^{4}$ \\ 'Department of Ophthalmology, Somalia \\ Mogadishu-Turkey Education and \\ Research Hospital, Mogadishu, Somalia; \\ ${ }^{2}$ Department of Ophthalmology, Antalya \\ Training and Research Hospital, Antalya, \\ Turkey; ${ }^{3}$ Department of Radiology, \\ Somalia Mogadishu - Turkey Education \\ and Research Hospital, Mogadishu, \\ Somalia; ${ }^{4}$ Department of Internal \\ Medicine, Somalia Mogadishu-Turkey \\ Education and Research Hospital, \\ Mogadishu, Somalia
}

Purpose: To evaluate the changes in intraocular pressure (IOP), central corneal thickness (CCT), axial length, and ocular surface in patients with end-stage renal failure after singlesession hemodialysis treatment and to examine the correlation of these findings with systemic hemodynamic parameters.

Patients and Methods: A total of 112 eyes of 112 patients enrolled in a hemodialysis treatment program for three times a week (approximately four hours per session) for at least three months in our hospital between December 2019 and March 2020 were included in the study. Approximately 30 minutes before and after hemodialysis, the best-corrected visual acuity measurement, slit-lamp examination, IOP measurement, and dilated fundus examination with the Goldmann applanation tonometer were performed in all patients. The axial length measurement was undertaken by ultrasonic biometry, and the CCT measurement by ultrasonic pachymetry. The ocular surface evaluation was performed based on the tear breakup time (BUT), basal secretion time (BST) and keratoepitheliopathy score. Blood pressure, serum osmolarity, and body weight were measured before and after hemodialysis. Results: The mean IOP decreased from $12.2 \pm 3.5$ to $10.8 \pm 2.2 \mathrm{mmHg}$, and the mean decrease was $1.4 \pm 2 \mathrm{mmHg}$, indicating statistical significance $(\mathrm{p}<0.001)$. The mean CCT dropped from $502 \pm 41.2$ to $494.1 \pm 35.4 \mu \mathrm{m}$, and the mean decrease was $8.1 \pm 7.7 \mu \mathrm{m}$, which also indicated a statistically significant change $(\mathrm{p}<0.001)$. The mean axial length was reduced from $23.1 \pm 0.8$ to $22.9 \pm 0.8 \mathrm{~mm}$. The reduction in the axial length was significant with an average value of $0.26 \pm 0.15 \mathrm{~mm}(\mathrm{p}<0.001)$. When the ocular surface changes were examined, BUT decreased from $7.4 \pm 3.1$ to $6.3 \pm 2.5 \mathrm{~s}$, BST from $8.6 \pm 1.7$ to $6.6 \pm 1.3 \mathrm{~mm}$, and the keratoepitheliopathy score increased from $0.36 \pm 0.61$ to $1.59 \pm 0.93$, and all these changes were statistically significant $(\mathrm{p}<0.001)$. There was a significant correlation between the axial length change and the IOP change $(r=0.202, p=0.03)$. A significant correlation was also observed between BST and plasma colloid osmotic pressure $(r=-0.268, p=0.004)$. In addition, there was a strong correlation between serum osmolarity and the keratoepitheliopathy score $(\mathrm{r}=-0.437, \mathrm{p}<0.001)$.

Conclusion: Hemodialysis causes significant changes in IOP, CCT, axial length, and ocular surface. These changes should be considered in the ophthalmological examination of patients after the hemodialysis session.

Keywords: end-stage renal failure, hemodialysis, axial length, intraocular pressure, Africa

\section{Introduction}

Chronic renal failure (CRF) is defined as the inability of the kidneys to maintain fluidelectrolyte balance, and chronic and progressive deterioration in metabolic-endocrine 
functions as a result of a decrease in the glomerular filtration value. ${ }^{1}$ Ocular pathologies can occur as a complication of systemic diseases, such as arterial hypertension and diabetes mellitus leading to CRF or due to CRF itself that results in hematological and metabolic disorders triggering such pathologies. ${ }^{2}$ Patients with CRF are usually treated using blood filtration devices, such as hemodialysis. ${ }^{3}$ Although hemodialysis is the main treatment of end-stage renal failure (ESRD), acute and chronic complications may occur after this treatment. ${ }^{4}$ Hypovolemia and increased osmotic pressure after hemodialysis may affect eye tissues with large amounts of blood flow.

Ocular effects associated with hemodialysis include changes in intraocular pressure (IOP), dry eye, conjunctival and perilimbal calcium deposits, band keratopathy, refractive changes, lenticular opacity and posterior subcapsular cataract, and ischemic optic neuropathy. ${ }^{5-7}$ The main goal of hemodialysis is to maintain body fluid balance. However, even after a single hemodialysis session, changes in systemic and ocular fluid volume can be observed and cause undesirable side effects.

Somalia is a low-income country in sub-Saharan Africa, bordering Kenya, Ethiopia, and Djibouti. Although previous studies have investigated the effect of ESRD and hemodialysis on ocular tissues, there is no study in the literature on ESRD patients receiving hemodialysis treatment in Somalia. In addition, since the effects of hemodialysis on ocular parameters are unclear, our prospective, cross-sectional study examines a population with short-term changes in ocular findings before and after a single hemodialysis session. This study aimed to determine the effects of hemodialysis on ocular surface, IOP, central corneal thickness (CCT), and axial length after a single hemodialysis session in patients in Somalia receiving this treatment due to ESRD, and to evaluate the correlation of these changes with systemic changes, namely body weight, mean arterial pressure, plasma colloid osmotic pressure, and serum osmolarity.

\section{Patients and Methods}

This hospital-based, prospective, cross-sectional study was carried out in Somalia Mogadishu-Turkey Training and Research Hospital after receiving approval from the ethical review committee of the hospital (decision number: 202 and MSTH/2904). The study was conducted by adhering to the principles of the Declaration of Helsinki. In addition, written and verbal informed consent was obtained from all participants.
According to our hypothesis, the short-term change in body fluids and content in patients undergoing hemodialysis session may cause changes in both intraocular pressure and axial length and dry eye. We planned our research to determine whether these changes were significant.

The study included 112 eyes of 112 patients enrolled in a hemodialysis treatment program for three times a week (approximately four hours per session) for at least three months in the hemodialysis unit of the abovementioned hospital between December 2019 and March 2020. The exclusion criteria were the presence of an active ocular infection, history of ocular surgery, history of ocular trauma, presence of glaucoma or ocular hypertension, active topical drug use, presence of pseudoexfoliative material, history of iridotomy, and corneal leukoma or cataract causing media opacity. The patients with retinal disease that would interfere with ocular examination and measurement, such as macular degeneration, proliferative diabetic retinopathy, vitreous hemorrhage, intraocular injection or laser therapy within 3 months of enrollment were excluded. In order to eliminate diurnal differences, only patients that underwent this treatment between 12:00 and 16:00 were included in the study. Ocular examinations were conducted were performed 30 minutes before and after the hemodialysis session (at 11:30 am and at 04:30 $\mathrm{pm})$. The left eyes of all patients were examined. The same type of hemodialysis device was used in all patients (Fresenius 4008S classix, Bad Homburg, Germany). The hemodialysis device was used with polysulfone membrane dialyzer and low molecular weight heparin as an anticoagulant. The bicarbonate in bipolar reverse osmosis water used with a dialysate flow rate of $500 \mathrm{~mL} / \mathrm{min}$ and blood flow rate of $200-400 \mathrm{~mL} / \mathrm{min}$. Dialysate temperature was 37 C. For all studies, the prescribed dialysate sodium concentration was set at $138 \mathrm{mmol} / \mathrm{l}$.

The best-corrected visual acuity (BCVA) measurement with the Snellen chart, slit-lamp examination, IOP measurement with the Goldmann applanation tonometer, and lastly, dilated fundus examination were performed $30 \mathrm{~min}-$ utes before and after the hemodialysis session. Spherical equivalent, as the representative of refractive status, was measured by an ophthalmologist using an automated refractometer (RK-F2, Canon, Tokyo, Japan). The ocular surface evaluation was performed based on the tear breakup time (BUT), basal secretion time (BST), and keratoepitheliopathy score.

For the BUT evaluation, the patient was placed in front of a biomicroscope (Righton MW50D, Tokyo, Japan), and 
sodium fluorescein strips were allowed to contact the lower fornix for a short period of time, and the cornea was stained through blinking. During the biomicroscopic examination, the patient was asked not to blink while a cobalt blue light was shined into the eye, and the time when the first black spots or lines formed in the tear layer on the cornea was recorded in seconds. This procedure was repeated three times, and the average of three measurements was determined as the BUT value. A BUT of less than 10 seconds was considered as abnormal.

For the BST evaluation, a drop of local anesthetic was instilled into the patient's eye, and the Schirmer paper of $5 \mathrm{~mm}$ width and $35 \mathrm{~mm}$ length with a bent end was inserted into the $1 / 3$ lateral of the lower lid and removed after $5 \mathrm{~min}$. The wetted length from the bent end was measured in $\mathrm{mm}$ and recorded. A tear secretion value of less than $5 \mathrm{~mm}$ was considered as abnormal.

Keratoepitheliopathy was evaluated based on the density and area of corneal staining with fluorescein. ${ }^{8}$ The severity of keratoepitheliopathy was obtained by multiplying the density and area scores, and evaluated as corneal surface damage index. The staining density was graded between 0 and 3 , where 0 indicated no punctate density, 1 rare density, 2 moderate density, and 3 high density. The staining area was also scored from 0 to 3 , with 0 indicating no staining, 1 indicating staining of less than one-third area, 2 indicating staining of more than one-third and covering two-thirds of the area, and 3 indicating staining that covered more than two-thirds of the area. ${ }^{9}$

IOP was measured by the same researcher (M.K.) under slit-lamp microscopy using the same Goldmann applanation tonometer (Haag-Streit, Switzerland), and the average of at least three measurements was taken. Full-circle $360^{\circ}$ gonioscopy was performed in all eyes with three mirror lenses. The CCT measurement was undertaken by ultrasonic pachymetry (Nidek US 4000 Echoscan, Gamagori, Japan). After a topical anesthetic was dropped into the eye, the patient was asked to look at a fixed target, and at least 10 measurements were taken from the central area with the tip of the probe perpendicular to the cornea. The average calculated by the measuring device was recorded as the CCT value. The axial length measurement was performed using an ultrasonic biometry device (Nidek US 4000 Echoscan, Gamagori, Japan). The average of at least five measurements was recorded as the axial length value. Lastly, one drop of $0.5 \%$ tropicamide (Tropamid®, Bilim Pharmaceuticals, Turkey) was applied to the eye for pupil dilatation.
Blood pressure, mean ocular perfusion pressure, serum osmolarity, and body weight were measured before and after hemodialysis. In addition, plasma total protein and albumin were obtained, and plasma colloid osmotic pressure was calculated before and after hemodialysis.

\section{Statistical Analysis}

The Statistical Package for the Social Sciences (SPSS v. 23.0, Chicago, USA) was used for the statistical analysis. Descriptive statistics; ie, mean \pm standard deviation (SD) values were used to describe quantitative data and frequencies and percentages for qualitative data. The Shapiro-Wilk test was used to determine the normality. Preoperative and postoperative data were analyzed using the paired $t$-test. The Pearson correlation test was used to identify possible correlations between the changes in eye findings and those in systemic hemodynamic parameters. This test was also used to determine whether there was a correlation between the axial length and IOP. A p value of $<0.05$ was considered statistically significant. Visual acuity was converted to the logarithm of the minimum resolution angle (logMAR) for the statistical analysis.

Every adult hemodialysis patient who fulfilled the inclusion criteria was included in this study. Sample size was calculated with $G^{*}$ Power 3.1.9.4 calculator power analysis with two sided exact one sample proportion case. The desired sample size at $5 \%$ level of significance, $80 \%$ power and the design effect of 0.108 is 112 .

\section{Results}

\section{Demographic and Etiological Data}

A total of 112 eyes of 112 patients (54.5\% male and $45.5 \%$ female) were included in the study. The mean age of the patients included in the study was $46.6 \pm 16.7$ years. The etiological causes of CRF in order of frequency were described in Table 1. The mean hemodialysis time was $26.3 \pm 17.7$ months (3-72 months).

\section{Effect of Hemodialysis on BCVA, IOP, CCT, and Ocular Surface}

When the eye findings were evaluated before and after hemodialysis, BCVA did not change significantly $(\mathrm{p}=$ 0.866). The mean IOP decreased from $12.2 \pm 3.5 \mathrm{mmHg}$ to $10.8 \pm 2.2 \mathrm{mmHg}$. The mean decrease in IOP $1.4 \pm 2$ $\mathrm{mmHg}$, which was statistically significant $(\mathrm{p}<0.001)$. The mean CCT dropped from $502 \pm 41.2 \mu \mathrm{m}$ to $494.1 \pm 35.4 \mu \mathrm{m}$, and the mean decrease was $8.1 \pm 7.7 \mu \mathrm{m}$, indicating 
Table I Distribution of Patients with Chronic Renal Failure According to Etiological Causes

\begin{tabular}{|l|l|}
\hline Etiology & $\begin{array}{l}\text { Number of } \\
\text { Patients (\%) }\end{array}$ \\
\hline Diabetes mellitus & $44(39.3)$ \\
Systemic hypertension & $26(23.2)$ \\
Primary glomerulonephritis & $12(10.7)$ \\
Interstitial nephritis/pyelonephritis & $11(9.8)$ \\
Secondary glomerulonephritis & $9(8)$ \\
Cystic, hereditary and congenital diseases of the kidney & $6(5.4)$ \\
Other causes & $4(3.6)$ \\
\hline
\end{tabular}

statistical significance $(\mathrm{p}<0.001)$. The mean axial length was reduced from $23.1 \pm 0.8 \mathrm{~mm}$ to $22.9 \pm 0.8 \mathrm{~mm}$, and the decrease in the axial length was significant with a mean value of $0.26 \pm 0.15 \mathrm{~mm}(\mathrm{p}<0.001)$. Examining the ocular surface changes, BUT decreased from $7.4 \pm 3.1 \mathrm{~s}$ to $6.3 \pm 2.5$ $\mathrm{s}$, BST from $8.6 \pm 1.7 \mathrm{~mm}$ to $6.6 \pm 1.3 \mathrm{~mm}$, and the keratoepitheliopathy score increased from $0.36 \pm 0.61$ to $1.59 \pm 0.93$, indicating that all three findings statistically significantly changed $(\mathrm{p}<0.001)$ (Table 2$)$. There was a significant correlation between the axial length change and the IOP change $(r=0.202, p=0.03)$ (Figure 1); however, no correlation was observed between the changes in CCT and IOP ( $p>0.05)$.

\section{Effect of Hemodialysis on Systemic Hemodynamic Parameters}

The mean value of serum osmolarity decreased from $345 \pm$ $31.8 \mathrm{mOsm} / \mathrm{L}$ to $326.3 \pm 32 \mathrm{mOsm}$. The mean decrease of $18.6 \pm 3 \mathrm{mOsm} / \mathrm{L}$ was statistically significant $(\mathrm{p}<0.001)$. Plasma colloid osmotic pressure increased from $25.3 \pm 1$ $\mathrm{mmHg}$ to $27.9 \pm 1.4 \mathrm{mmHg}$, and the difference of $2.5 \pm 0.4$ $\mathrm{mmHg}$ was significant $(\mathrm{p}<0.001)$. The mean arterial blood pressure change was significant with a decrease of $11.8 \pm 5$ $\mathrm{mmHg}(\mathrm{p}<0.001)$. The change in the mean body weight was $1.2 \pm 2.4 \mathrm{~kg}$, which was also statistically significant $(\mathrm{p}<0.001)$ (Table 3). When the changes in hemodynamic parameters and those in eye parameters were correlated individually, there was no statistical significance in any value $(\mathrm{p}>0.05)$. However, there was a significant correlation between BST and plasma colloid osmotic pressure $(r=-0.268, p=0.004)$. In addition, a strong correlation was observed between serum osmolarity and the keratoepitheliopathy score $(\mathrm{r}=-0.437, \mathrm{p}<0.001)$.

\section{Discussion}

In this study, we investigated the effect of short-term hemodialysis treatment on IOP, CCT, axial length, and ocular surface, and the relationship of these changes with ocular and systemic parameters. After single-session hemodialysis treatment, IOP, CCT, axial length, BUT, and BST decreased whereas the keratoepitheliopathy score was increased. In addition, serum osmolarity, blood pressure, and body weight decreased, and plasma colloid osmotic pressure increased. According to the correlation analysis, there was a significant correlation between BST and plasma colloid osmotic pressure and between the keratoepitheliopathy score and serum osmolarity, but the remaining ocular and systemic parameters were not correlated.

The basic principle of hemodialysis is the removal of osmotic active substances by diffusion and the reduction of blood osmolarity through body fluid loss. These two effects are closely related to IOP. Dehydration decreases IOP while reducing blood osmolarity increases IOP. However, the net effect on IOP varies in different studies. The effects of hemodialysis on IOP changes remain uncertain. In 2015, Liakopoulos et al ${ }^{10}$ published a review and

Table 2 Effect of Hemodialysis on BCVA, IOP, CCT, Axial Length, and Ocular Surface in Patients with Chronic Renal Failure

\begin{tabular}{|l|l|l|l|l|}
\hline Variable & Before Hemodialysis Mean \pm SD & After Hemodialysis Mean \pm SD & Difference Mean \pm SD & P value \\
\hline BCVA (LogMAR) & $0.49 \pm 0.42$ & $0.49 \pm 0.42$ & $0 \pm 0.03$ & 0.866 \\
Spherical equivalent, (D) & $-0.43 \pm 0.26$ & $-0.45 \pm 0.22$ & $-0.02 \pm 0.04$ & 0.524 \\
IOP (mmHg) & $12.2 \pm 3.5$ & $10.8 \pm 2.2$ & $1.4 \pm 2$ & $<0.001$ \\
CCT $(\mu \mathrm{m})$ & $502 \pm 41.2$ & $494.1 \pm 35.4$ & $8.1 \pm 7.7$ & $<0.001$ \\
Axial length (mm) & $23.1 \pm 0.8$ & $22.9 \pm 0.8$ & $0.26 \pm 0.15$ & $<0.001$ \\
BUT (sec) & $7.4 \pm 3.1$ & $6.3 \pm 2.5$ & $1 \pm 0.9$ & $<0.001$ \\
BST (mm) & $8.6 \pm 1.7$ & $6.6 \pm 1.3$ & $2 \pm 0.7$ & $<0.001$ \\
Keratoepitheliopathy score & $0.36 \pm 0.61$ & $1.59 \pm 0.93$ & $-1.2 \pm 0.7$ & $<0.001$ \\
\hline
\end{tabular}

Abbreviations: BCVA, best-corrected visual acuity; D, dioptry; IOP, intraocular pressure; CCT, central corneal thickness; SD, standard deviation; BUT, tear breakup time; BST, basal secretion time. 


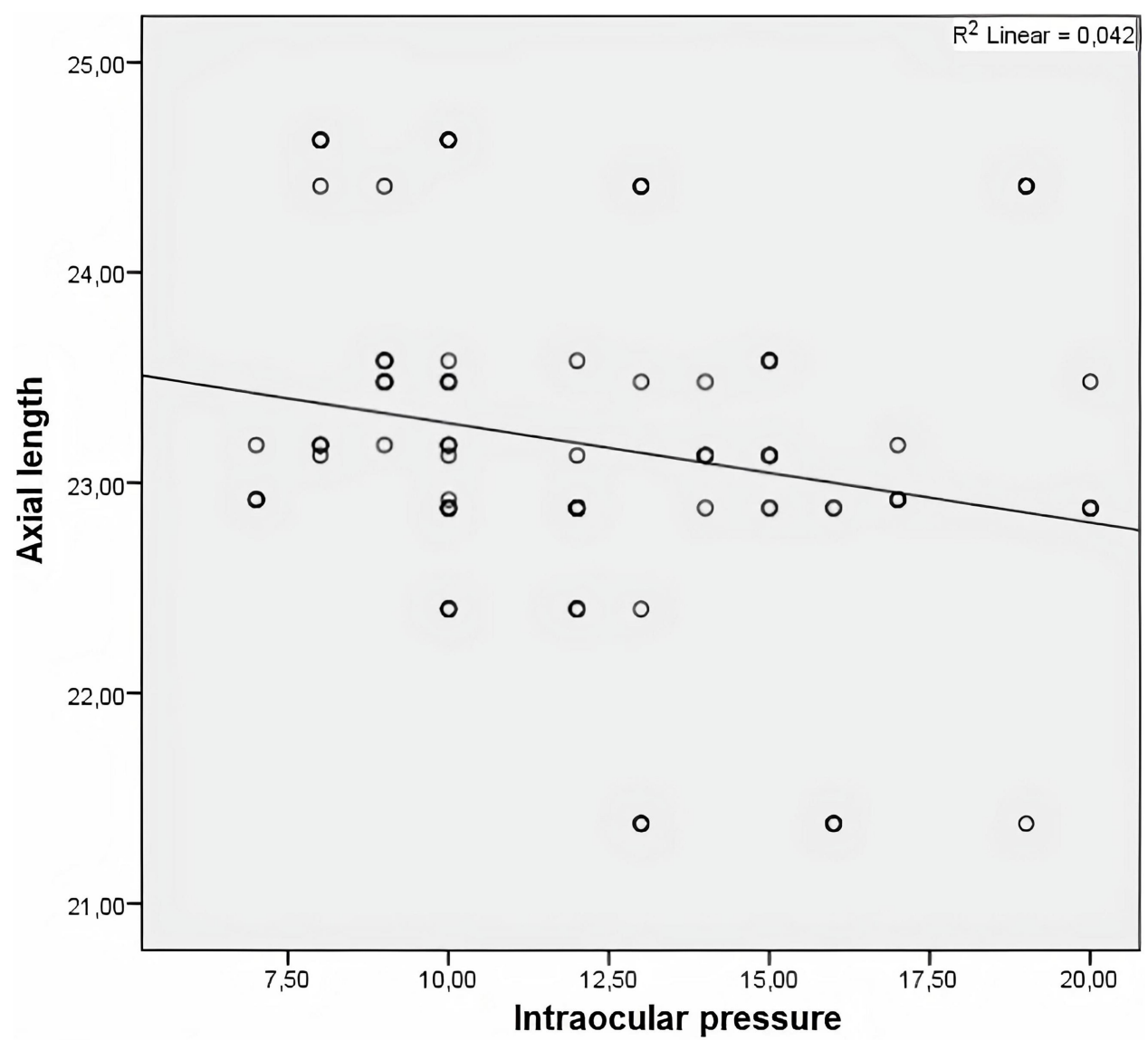

Figure I Correlation between axial length and intraocular pressure.

discussed the changes in IOP in relation to hemodialysis in three groups: those with increased IOP, those with reduced IOP, and those without any change in IOP. Accordingly, the authors found that while the first studies had been carried out with longer and less effective hemodialysis systems, the development of more effective dialysis membranes and devices in recent years significantly prevented increases in IOP. Recent studies indicate that IOP is decreased or does not change after hemodialysis. ${ }^{4,11,12}$ The results of our study are also consistent with those of previous studies. The reason for no increase in IOP in these studies has been linked to the use of advanced hemodialysis techniques. Cağlayan et $\mathrm{al}^{4}$ suggested that after hemodialysis, total body fluid loss and increased plasma colloid osmotic pressure caused a water flow from the ocular region to the plasma, which led to a decrease in IOP. Yang et $\mathrm{al}^{13}$ reported that the decrease in IOP was related to the relationship between the mean plasma albumin level and the ultrafiltration rate. Increased oncotic pressure caused by ultrafiltration causes a decrease in IOP after dialysis. The change in IOP is higher in cases with an increased albumin level and ultrafiltration degree.

Table 3 Effect of Hemodialysis on Systemic Hemodynamic Parameters in Patients with Chronic Renal Failure

\begin{tabular}{|l|l|l|l|l|}
\hline Variable & $\begin{array}{l}\text { Before Hemodialysis } \\
\text { Mean } \pm \text { SD }\end{array}$ & $\begin{array}{l}\text { After Hemodialysis } \\
\text { Mean } \pm \text { SD }\end{array}$ & Difference Mean \pm SD & P value \\
\hline Serum osmolarity (mOsm/L) & $345 \pm 31.8$ & $326.3 \pm 32$ & $18.6 \pm 3$ & $<0.00 \mathrm{I}$ \\
Plasma colloid osmotic pressure $(\mathrm{mmHg})$ & $25.3 \pm 1$ & $27.9 \pm 1.4$ & $-2.5 \pm 0.4$ & $<0.00 \mathrm{I}$ \\
MABP (mmHg) & $120.6 \pm 29.4$ & $108.7 \pm 25.4$ & $11.8 \pm 5$ & $<0.00 \mathrm{I}$ \\
MOPP & 49.2 & 44.3 & $-4.9 \pm 13.7$ & $<0.00 \mathrm{I}$ \\
Body weight $(\mathrm{kg})$ & $60.8 \pm 7.1$ & $59.5 \pm 6.7$ & $1.2 \pm 2.4$ & $<0.00 \mathrm{I}$ \\
\hline
\end{tabular}

Abbreviations: MABP, mean arterial blood pressure; MOPP, mean ocular perfusion pressure. 
From this perspective, the results of our study support the idea that the decrease in IOP is caused by the movement of intraocular fluid from the eye to plasma after hemodialysis.

Tropicamide eye drop, which we use for pupil dilation before hemodialysis, has a short-acting parasympatholytic drug. Inhibition of the parasympathetic constrictor muscle in the iris results in dilation of the pupil because the action of sympathetic dilator muscles is unopposed. Tropicamide eye drops are known to cause an increase in intraocular pressure. ${ }^{14}$ Tropicamide has an average of 4 hours of action. Considering that the average duration of hemodialysis after the application of tropicamide eye drops to the patients included in the study was 4 hours and that the patients were kept for an average of 30 minutes for examination after hemodialysis, it can be suggested that tropicamide had no effect on the measurement results.

It is similarly believed that there is a possible decrease in the corneal thickness and axial length due to fluid loss during hemodialysis. The results of the current study showed a marked decrease in CCT after hemodialysis with an average change of $8.1 \pm 7.7 \mu \mathrm{m}$. In addition, there was a decrease of $0.26 \pm 0.15 \mathrm{~mm}$ in the mean axial length. The decrease in both of these values was statistically significant. Furthermore, there was a significant correlation between the axial length change and the IOP change. However, the changes in neither CCT nor the axial length were correlated with any systemic changes, including body fluid loss. Although the change in CCT in ESRD patients receiving hemodialysis treatment has been investigated in many studies, there is very limited research exploring the change in the axial length and its correlation with systemic parameters. In a recent study conducted in 2018 , Wang et al ${ }^{15}$ stated that the vitreous axial length increased after hemodialysis in patients who received this treatment due to CRF. The researchers suggested that the increase in the vitreous axial length caused the lens to swell with more fluid as a result of the imbalance in the urea nitrogen level formed between the aqueous humor and the lens, thereby increasing the axial length. They also suggested that the fluid in the vitreous increased as a result of low plasma osmolarity, and this mechanism may have also caused an increase in the axial length of the vitreous body. However, that study did not contain any data on the total axial diameter. Gracitelli et $\mathrm{al}^{16}$ stated that there was no significant change in the axial length during hemodialysis treatment. Çalışkan et al ${ }^{17}$ found that the axial length increased only very slightly after the hemodialysis session and suggested that this increase could be due to the decreased fluid volume of the choroid and retina after the hemodialysis session. In contrast to these findings, the results of our study show that the axial length significantly decreased after the hemodialysis session. We consider that not only the decreased fluid volume in the retina and choroid but also the decreased anterior chamber volume and corneal thickness were effective in this finding. This hypothesis is supported by the presence of a significant decrease in CCT and a significant correlation between the axial length and IOP in our study. In previous studies conducted with patients receiving hemodialysis due to ESRD, CCT was found to significantly decrease after the treatment session., ${ }^{3,4,18}$ A decrease in body fluid volume and an increase in plasma colloid osmotic pressure after hemodialysis causes a fluid flow from the eye to the plasma, resulting in a decrease in the corneal thickness. ${ }^{3}$ However, the decrease in IOP, which was observed to be significant in our study, may also be related to the decrease in the corneal thickness since the IOP values measured by Goldmann applanation tonometry depend on CCT.

In our study, ocular surface changes were evaluated using BUT, BST (Schirmer's test), and keratoepitheliopathy score, and there were significant changes in the results of all three tests after a single hemodialysis session. In the correlation analysis, a significant association was found between BST and plasma colloid osmotic pressure, and between serum osmolarity and the keratoepitheliopathy score. The decrease in both BUT and BST values after the hemodialysis session suggests reduced tear production and deteriorated stability of the tear film layer. In addition, the increase in the keratoepitheliopathy score after the hemodialysis session may be an indicator of increased inflammation in tears. After hemodialysis treatment, tear production decreases due to dehydration. ${ }^{6}$ In addition, increased urea level in tears causes an increase in tear hyperosmolarity, ${ }^{3}$ which induces inflammatory cytokines and matrix metalloproteases on the epithelial cell surface. This results in cell apoptosis, ocular surface damage, goblet cell damage, and tear film instability. ${ }^{19}$ There are a limited number of studies investigating ocular surface changes in patients receiving hemodialysis for ESRD. In 2013, Jung et $\mathrm{al}^{3}$ showed that after single-session hemodialysis, BUT and Schirmer's test results decreased and the keratoepitheliopathy score increased, which is consistent with our findings related to ocular surface changes. 
The strengths of our study are that we had a high number of participants, and our study is the first to reveal the ocular and systemic parameters of patients receiving hemodialysis treatment due to ESRD in North Africa and Somalia.

The limitations of the study include ocular measurements not being measured during the hemodialysis session and the absence of a control group. Another limitation is the timing difference between ocular and systemic assessments. Systemic parameters were measured just before and after the hemodialysis session, while ocular parameters were measured 30 minutes before the hemodialysis session. It is unclear whether there has been a change in both systemic and ocular parameters during this time.

In conclusion, ophthalmological findings, namely IOP, CCT, axial length, and ocular surface changes were consistent and significant after a single hemodialysis session in patients receiving this treatment due to ESRD. During the ophthalmologic examination of patients undergoing hemodialysis treatment, it should be kept in mind that there can be more dry eye findings than in the normal population, and this situation becomes even more severe after the hemodialysis session. The low levels of IOP and CCT after the hemodialysis session indicates the necessity of undertaking a careful eye examination on the same day. In these patients, it seems more appropriate to evaluate IOP before or after the hemodialysis session. In addition, be careful of measuring axial length because it changes with hemodialysis.

\section{Abbreviations}

$\mathrm{CRF}$, chronic renal failure; ESRD, end-stage renal disease; IOP, intraocular pressure; CCT, central corneal thickness; BUT, tear breakup time; BST, basal secretion time.

\section{Ethical Approval}

Approval was obtained from the ethical review committee of Somalia Mogadishu-Turkey Education and Research Hospital (date: December 26, 2019, decision number: 202, MSTH/2904).

\section{Consent}

Written informed consent was obtained from the patients before any examination or treatment was performed.

\section{Author Contributions}

All authors made substantial contributions to the conception and design, acquisition of data, or analysis and interpretation of data; took part in drafting the article or revising it critically for important intellectual content; gave final approval of the version to be published; and agree to be accountable for all aspects of the work.

\section{Funding}

No funding or financial support was received for this study.

\section{Disclosure}

The authors report no conflict of interest in this work. The authors declare that they have no known competing financial interests or personal relationships that could have influenced the work reported in this paper. The authors have no financial interest or relationship to disclose.

\section{References}

1. Webster AC, Nagler EV, Morton RL, et al. Chronic kidney disease. Lancet. 2017;389(10075):1238-1252. doi:10.1016/S0140-6736(16) 32064-5

2. Minen M, Kemak Arici M, Erdogan H, Topalkara A, Toker IGF. Eye findings in haemodialysis patients. Turk J Ophthalmol. 2001;31:352-360.

3. Jung JW, Yoon MH, Lee SW, et al. Effect of hemodialysis (HD) on intraocular pressure, ocular surface, and macular change in patients with chronic renal failure: effect of hemodialysis on the ophthalmologic findings. Graefe's Arch Clin Exp Ophthalmol. 2013;251 (1):153-162. doi:10.1007/s00417-012-2032-6

4. Caglayan M, Kosekahya P, Takmaz T, et al. Effects of hemodialysis on corneal and anterior chamber morphometry and intraocular pressure in patients with end-stage renal disease. Arq Bras Oftalmol. 2017;80(3):176-180. doi:10.5935/0004-2749.20170043

5. Aktas Z, Ozdek S, Asli Dinc U, et al. Alterations in ocular surface and corneal thickness in relation to metabolic control in patients with chronic renal failure. Nephrology. 2007;12(4):380-385. doi:10.1111/ j.1440-1797.2007.00805.x

6. Charlton JF, Schwab IR, Stuchell R. Tear hyperosmolarity in renal dialysis patients asymptomatic for dry eye. Cornea. 1996;15 (4):335-339. doi:10.1097/00003226-199607000-00001

7. Mullaem G, Rosner MH. Ocular problems in the patient with end-stage renal disease. Semin Dial. 2012;25(4):403-407. doi:10. 1111/j.1525-139X.2012.01098.x

8. Yoon KC, Song B-Y, Seo MS. Effects of smoking on tear film and ocular surface. Korean J Ophthalmol. 2005;19(1):18. doi:10.3341/ kjo.2005.19.1.18

9. Yoon KC, Im SK, Seo MS. Changes of tear film and ocular surface in diabetes mellitus. Korean $J$ Ophthalmol. 2004;18(2):168-174. doi:10.3341/kjo.2004.18.2.168

10. Liakopoulos V, Demirtzi P, Mikropoulos DG, et al. Intraocular pressure changes during hemodialysis. Int Urol Nephrol. 2015;47 (10):1685-1690. doi:10.1007/s11255-015-1043-8

11. Kilavuzoglu AEB, Yurteri G, Guven N, et al. The effect of hemodialysis on intraocular pressure. Adv Clin Exp Med. 2018;27 (1):105-110. doi:10.17219/acem/68234

12. Yuksel N, Duru N, Uz E, et al. Evaluation of intraocular pressure by ocular response analyzer in patients undergoing hemodialysis. $J$ Glaucoma. 2016;25(4):e355-e358. doi:10.1097/IJG.0000000000000364

13. Yang SJ, Han YH, I Song G, et al. Changes of choroidal thickness, intraocular pressure and other optical coherence tomographic parameters after haemodialysis. Clin Exp Optom. 2013;96(5):494-499. doi:10.1111/cxo.12056 
14. Hong D, Tripathy K Tropicamide. 2020. Available from: http://www. ncbi.nlm.nih.gov/pubmed/31082113. Accessed November 2, 2020.

15. Wang L, Yin G, Yu Z, et al. Effect of hemodialysis on eye coats, axial length, and ocular perfusion pressure in patients with chronic renal failure. J Ophthalmol. 2018;2018:1-6.

16. Gracitelli CPB, Stefanini FR, Penha F, et al. Anterior chamber depth during hemodialysis. Clin Ophthalmol. 2013;7:1635-1639. doi:10.2147/OPTH.S45952

17. Calıskan S, Celikay O, Bicer T, et al. Effect of hemodialysis on intraocular lens power calculation. Ren Fail. 2016;38(2):209-213. doi:10.3109/0886022X.2015.1128235
18. Dinc UA, Ozdek S, Aktas Z, et al. Changes in intraocular pressure, and corneal and retinal nerve fiber layer thickness during hemodialysis. Int Ophthalmol. 2010;30(4):337-340. doi:10.1007/ s10792-009-9339-2

19. Li DQ, Chen Z, Song XJ, et al. Stimulation of matrix metalloproteinases by hyperosmolarity via a JNK pathway in human corneal epithelial cells. Invest Ophthalmol Vis Sci. 2004;45(12):4302. doi:10.1167/iovs.04-0299

\section{Publish your work in this journal}

The International Journal of General Medicine is an international, peer-reviewed open-access journal that focuses on general and internal medicine, pathogenesis, epidemiology, diagnosis, monitoring and treatment protocols. The journal is characterized by the rapid reporting of reviews, original research and clinical studies across all disease areas. The manuscript management system is completely online and includes a very quick and fair peer-review system, which is all easy to use. Visit http://www.dovepress.com/ testimonials.php to read real quotes from published authors. 